Archives de sciences sociales des religions

109 | janvier-mars 2000

Formes religieuses caractéristiques de

l'ultramodernité : France, Pays-Bas, États-Unis, Japon, analyses globales

\title{
Les attitudes religieuses en France : quelles recompositions en cours?
}

\author{
Pierre Bréchon
}

\section{(2) OpenEdition \\ Journals}

Édition électronique

URL : http://journals.openedition.org/assr/20165

DOI : 10.4000/assr.20165

ISSN : $1777-5825$

Éditeur

Éditions de l'EHESS

Édition imprimée

Date de publication : 1 mars 2000

Pagination : 11-30

ISBN : 2-222-96690-6

ISSN : 0335-5985

Référence électronique

Pierre Bréchon, «Les attitudes religieuses en France : quelles recompositions en cours? ", Archives de sciences sociales des religions [En ligne], 109 | janvier-mars 2000, mis en ligne le 19 août 2009,

consulté le 19 avril 2019. URL : http://journals.openedition.org/assr/20165 ; DOI : 10.4000/assr.20165

Ce document a été généré automatiquement le 19 avril 2019

(C) Archives de sciences sociales des religions 


\title{
Les attitudes religieuses en France: quelles recompositions en cours?
}

\author{
Pierre Bréchon
}

1 Le paysage religieux de la France a incontestablement beaucoup changé au cours du XX siècle $^{1}$. La prise en compte du temps long facilite la lecture des évolutions les plus massives mais fait courir le risque des interprétations évolutionnistes, comme si tous les changements allaient dans le même sens en longue période. Lorsqu'on considère le panorama du moment et les évolutions de très courte période, on voit mieux les tensions et les évolutions de sens contraire qui se déroulent en même temps. Le temps court sera ici privilégié pour tester des hypothèses classiques. Comment a évolué ces dernières années le paysage religieux ? Peut-on parler d'une sécularisation de la société ? Si tel est le cas, quelle est la nature de cette sécularisation? Simple perte d'emprise de la religion dominante? Déclin des certitudes religieuses au profit de croyances possibles, du doute, de l'indifférence religieuse, de la montée d'un athéisme tranquille ou militant? Peut-on parler de retour du religieux comme certains l'ont prétendu ou assiste-t-on simplement à des formes de recompositions et de bricolages des croyances dans un univers religieux moins institutionnel et plus sécularisé?

2 Ces questions seront traitées en utilisant une enquête récente de sociologie des religions, réalisée par une fédération internationale de chercheurs, l'International Social Survey Programme (ISSP2). La France n'a rejoint que depuis 1996 ce programme international ${ }^{3}$. Comme elle ne faisait pas partie du programme en 1991, date d'une précédente enquête religieuse avec un questionnaire en large partie identique, les résultats de 1998 ne peuvent donc pas être comparés avec l'enquête antérieure. Quelques comparaisons sont cependant possibles avec l'enquête Valeurs de $1990^{4}$.

\section{Une image des Églises en demi-teinte}

3 L'image des Églises est un élément important de leur impact social. Si l'on valorise une institution, considérée comme très utile, on aura tendance à écouter ses messages. Si l'on 
juge au contraire une organisation comme peu crédible, démodée, inutile voire nuisible, on ne prendra pas au sérieux ses déclarations. Si enfin un groupe religieux n'a pas d'image dans l'opinion parce qu'il est méconnu, il ne peut bien sûr avoir une influence. Plusieurs questions permettent d'apprécier l'image des groupes religieux considérés globalement.

On sait qu'aujourd'hui l'image des grandes institutions politiques, culturelles et sociales n'est souvent pas très bonne. Tous les médiateurs institutionnels du rapport des individus à l'ordre social sont facilement soupçonnés de travailler pour leur propre compte plus que pour leurs mandants. La mesure de la confiance faite à un ensemble d'institutions, dont les Églises, montre que les Français leur font une confiance relative. $47 \%$ disent avoir au moins une certaine confiance, pourcentage plus élevé que pour le Parlement ( $43 \%$ ) ou surtout les entreprises et le monde des affaires ( $31 \%$ mais plus bas que pour la justice $(61 \%)$ et le système scolaire $(78 \%)$. Au vu de deux autres questions, l'image des religions apparaît même plus négative. Deux tiers des Français pensent que « de par le monde, les religions apportent plus de conflit que de paix ». Deux tiers des répondants trouvent aussi que « les gens qui ont de fortes convictions religieuses sont souvent trop intolérants envers les autres ». Les religions seraient donc potentiellement dangereuses comme toute organisation trop sûre d'elle-même, trop liée à la défense d'une idéologie.

Si l'image des religions apparait globalement assez négative, il y a cependant un indicateur dont les résultats sont plus positifs. Il porte sur le degré de vérité des religions : si $23 \%$ «trouvent très peu de vérité dans les religions », $52 \%$ au contraire «trouvent des vérités fondamentales dans beaucoup de religions » et $6 \%$ ne « trouvent la vérité que dans une seule religion ${ }^{5} »$. Le premier item exprime une position laïciste de critique de toutes les formes religieuses, le dernier représente la position religieuse fondamentaliste. L'image des religions est donc ici plutôt positive puisqu'il n'y a qu'environ un quart de réponses négatives. On peut penser que. pour beaucoup de ceux qui choisissent l'item intermédiaire, les grandes religions sont globalement considérées comme vecteur d'humanisme, elles transmettent les valeurs de l'humanité et sont des voies d'accès à la sagesse. C'est en cela qu'elles sont porteuses de vérités fondamentales.

Ceci dit, ceux qui pensent qu'il y a des vérités fondamentales dans toutes les religions croient en même temps assez souvent qu'elles peuvent être facteur de conflit. L'image des religions est souvent mêlée pour chaque individu. Pour beaucoup, il faut « en prendre et en laisser » dans le discours des religions, on peut faire son profit des vérités qu'elles proposent, mais elles absolutisent leur part de vérité et deviennent ainsi potentiellement dangereuses. L'intolérance guette toute religion dès qu'elle se prétend voie exclusive d'accès à la vérité. Autant l'exclusivisme et l'absolutisation des discours religieux sont condamnés par beaucoup, autant l'ouverture sur des traditions religieuses diversifiées est considérée positivement. L'accès possible à différentes religions est perçu comme une source de richesse dans un univers individualisé où chacun veut se construire son identité et ses référents sans avoir à les trouver dans une tradition toute faite. La sympathie est forte lorsque les religions fonctionnent comme des repères offerts à la libre décision de chacun et comme des guides possibles pour la vie personnelle, par contre l'antipathie devient tout aussi forte lorsque les groupes religieux sont perçus comme des carcans et des chemins imposés.

7 Bien sûr, l'image des religions que l'on vient de brosser à partir de l'enquête ISSP reste très globale. Chaque groupe religieux peut avoir sa propre image, éventuellement très décalée par rapport à cette image moyenne des Églises. Quelques références à des 
sondages passés le montreront. Ainsi, une question mesurant le " palmarès des religions ${ }^{6}$ " indique que le catholicisme est apprécié par les deux tiers des Français, le protestantisme par la moitié, l'orthodoxie par le quart, le judaïsme et le bouddhisme par environ $15 \%$ et l'islam par seulement $6 \%$. Certaines religions sont probablement peu citées parce que mal connues. Mais ceci ne suffit pas à expliquer le très bas niveau de l'islam qui est probablement craint en tant que système d'emprise sur la culture, voire la politique. Au niveau des principes, il n'y a pas de fort rejet de l'islam comme foi personnelle et privée : $61 \%$ estiment que « la foi islamique est aussi respectable que la foi chrétienne ${ }^{7}$ ». Par contre beaucoup ne tolèrent pas que la différence islamique puisse se dire dans l'espace public français : $63 \%$ sont partisans d'interdire totalement le foulard islamique à l'écoles.

8 En ce qui concerne la religion catholique, si son image semble être plutôt positive, d'autres données introduisent des nuances. Si des majorités de Français la disent ouverte sur le monde (62\%) et tolérante ( $53 \%$ ), seulement $36 \%$ estiment qu'elle a su s'adapter au monde moderne ${ }^{9}$. L'image du pape reste plutôt positive mais moins nettement qu'autrefois et son image est même devenue médiocre chez les jeunes ${ }^{10}$. Le discours du catholicisme est considéré par de fortes majorités comme illégitime sur toutes les questions de morale et de sexualité (l'Église n'a pas à réguler la vie privée) ainsi que sur les problèmes proprement politiques. Ce n'est que sur certains problèmes sociaux, tout particulièrement sur l'aide au Tiers-monde, que son discours est jugé légitime ${ }^{11}$. Cette reconnaissance de son rôle humanitaire explique d'ailleurs la bonne image des missionnaires ${ }^{12}$.

9 L'analyse statistique des questions ISSP montre que l'image des Églises est bien meilleure chez les personnes qui se sentent elles-mêmes religieuses, qui sont bien intégrées au catholicisme et qui croient en Dieu. Ainsi, les catholiques pratiquants font presque tous confiance aux Églises et aucun ne pense qu'elles comportent très peu de vérité. Mais ils sont $72 \%$ à choisir la vision tolérante (des vérités dans beaucoup de religions) et seulement $22 \%$ l'item fondamentaliste (des vérités seulement dans la mienne). Par ailleurs, environ un quart des catholiques pratiquants reconnaît que les religions apportent souvent plus de conflit que de paix. Ils savent même prendre leurs distances par rapport à des positions de leur propre Église ${ }^{13}$. Pour beaucoup, la foi catholique n'est plus un absolu non critiquable et la seule source de vérité. Voilà un trait de l'ouverture des catholiques français à la culture de l'autonomie individuelle ${ }^{14}$.

10 L'identité religieuse des individus influence fortement leurs jugements sur les religions. Dans un pays en voie de sécularisation, il est donc très difficile pour les Églises d'avoir une très bonne image. Au vu des images en demi-teinte qui se dégagent des réponses, l'emprise des religions sur la société française apparaît plutôt faible. L'opinion n'idéalise pas les religions. Elle fait preuve de beaucoup de recul critique. La sécularisation de la société française est cependant loin d'être complète puisqu'il existe des images construites des principaux groupes religieux et que les religions gardent une image en demi-teinte, ni franchement hostile, ni franchement favorable. 


\section{Un catholicisme affaibli mais qui reste la religion dominante}

11 On peut avoir le sentiment que le panorama religieux global n'a pas beaucoup bougé dans la dernière décennie. Alors que $62 \%$ des Français disaient en 1990 appartenir à une religion, on n'en retrouve plus que $55 \%$.en 1998. Le pourcentage de catholiques passe dans le même temps de 57 à $51 \%$ alors que le pourcentage de sans religion monte de 39 à $45 \%$. Cette évolution s'explique essentiellement par le renouvellement des générations. Les jeunes générations étant beaucoup moins religieuses et intégrées au catholicisme que les plus vieilles (cf. la ventilation par âge donnée dans le tableau 1), au fur et à mesure que les générations âgées sont remplacées par des plus jeunes, de manière progressive mais nette, les taux de catholiques déclarés décroissent et les sans religion montent. Depuis 10 ans, on observe donc essentiellement le prolongement des tendances antérieures, des évolutions lentes mais régulières, lourdes et de long terme. Et il est clair que, parmi les catholiques français, beaucoup sont aujourd'hui très détachés de leur institution religieuse de référence puisque plus de la moitié des gens qui se déclarent catholiques ne pratiquent jamais. Les expressions employées par Jean-Paul Willaime pour désigner la France, "un pays catholique de culture laïque » et/ou "un pays laïque de culture catholique » apparaissent donc tout à fait adéquates ${ }^{15}$. Près de la moitié de la population affirme clairement sa distance d'avec toute religion, un gros quart continue de se dire catholique mais est de fait détaché. Les valeurs séculières dominent et le déclin du catholicisme n'a pas été véritablement remplacé par la montée d'autres univers religieux. Certes, les religions minoritaires se développent mais le catholicisme affaibli reste la principale religion structurant l'identité religieuse des Français. Dans l'enquête, les autres religions ne représentent que $4 \%$ de l'échantillon. Même si ce pourcentage sous-estime la réalité ${ }^{16}$, le pluralisme religieux reste en France un phénomène quantitativement limité. Cette remarque, qui peut paraître banale, doit néanmoins être rappelée, tant les observateurs médiatiques et parfois sociologiques ne voient que les phénomènes minoritaires, considérés par principe comme initiateurs du futur et souvent indûment majorés ${ }^{17}$.

Tableau 1 - Intégration au catholicisme selon l'âge (\% verticaux)

\begin{tabular}{|c|c|c|c|c|c|c|c|}
\hline Catholique & $\begin{array}{c}18-24 \\
\text { ans }\end{array}$ & $\begin{array}{c}25-34 \\
\text { ans }\end{array}$ & $\begin{array}{c}35-49 \\
\text { ans }\end{array}$ & $\begin{array}{c}50-64 \\
\text { ans }\end{array}$ & $\begin{array}{c}65 \text { ans } \\
\text { et }+\end{array}$ & $\begin{array}{c}\text { Ensemble } \\
1998\end{array}$ & $\begin{array}{c}\text { Rappel } \\
\text { Valeurs } 90\end{array}$ \\
\hline Pratiquant régulier $(n=127)$ & 3 & 3 & 4 & 13 & 34 & 12 & 15 \\
\hline Pratiquant irregulier ( $\mathrm{n}=142$ ) & 11 & 8 & 16 & 12 & 16 & 13 & 14 \\
\hline Non pratiquant $(\mathrm{n}=293)$ & 18 & 34 & 25 & 32 & 20 & 27 & 28 \\
\hline Sans religion $(n=489)$ & 63 & 51 & 50 & 37 & 27 & 45 & 39 \\
\hline
\end{tabular}

Conformément à ce qu'on trouve dans toutes les enquêtes de sociologie des religions, les indicateurs de pratique sont très liés entre eux. Le tableau 2 montre que l'intégration au catholicisme, mesurée par le critère de l'assistance à la messe, est très liée à la participation à des activités religieuses ${ }^{18}\left(\mathrm{~V}\right.$ de $\left.\mathrm{Cramer}^{19}=0.49\right)$ et à l'intensité de la prière ( $\mathrm{V}$ de Cramer $=0.44^{20}$ ). Ce dernier résultat montre une forte congruence entre une pratique ritualisée et collective et une pratique religieuse privée. Lorsqu'on va régulièrement à la messe, on a aussi toutes chances de prier souvent individuellement. 
Alors que si l'on est sans religion, il est fort rare que l'on prie souvent. L'hypothèse parfois émise, selon laquelle la religion s'effiloche dans sa dimension collective mais se maintient dans sa dimension privée, ne semble pas confirmée: on n'observe pas un découplage entre pratique collective et privée.

Tableau 2 - Intensité des activités religieuses et de la prière selon le degré d'intégration au catholicisme (\% verticaux)

\begin{tabular}{|l|rrr|r|r|}
\hline & \multicolumn{3}{|c|}{ Catholique } & \multirow{2}{*}{$\begin{array}{c}\text { Sans } \\
\text { religion }\end{array}$} & Ensemble \\
\cline { 2 - 3 } & $\begin{array}{c}\text { Pratiquant } \\
\text { régulier }\end{array}$ & $\begin{array}{c}\text { Pratiquant } \\
\text { irrégulier }\end{array}$ & $\begin{array}{c}\text { Non } \\
\text { pratiquant }\end{array}$ & \\
\hline Activités religieuses & 12 & 29 & 66 & 90 & 65 \\
- jamais & 21 & 33 & 28 & 8 & 19 \\
- une ou deux fois par an & 67 & 38 & 6 & 2 & 17 \\
- plus souvent & & & & & \\
\hline Prière & 0 & 3 & 26 & 78 & 43 \\
- jamais & 1 & 1 & 25 & 9 & 12 \\
- 1 ou 2 fois par an & 2 & 23 & 20 & 5 & 11 \\
- plusieurs fois par an & 17 & 39 & 12 & 4 & 12 \\
- 1 à 4 fois par mois & 81 & 34 & 17 & 4 & 22 \\
\hline
\end{tabular}

L'échelle d'intégration au catholicisme est aussi très liée au sentiment d'être religieux ${ }^{21}$ ( $\mathrm{V}$ de Cramer $=0.57$ ). Plus on se sent religieux, plus on est intégré au catholicisme. Presque tous ceux qui se disent religieux se déclarent aussi catholiques ${ }^{22}$, même si leur appartenance est souvent vague. S'il y a un certain développement du pluralisme religieux en France, s'il y a aussi une prise de distance des individus par rapport aux religions instituées, il n'y a pas actuellement une véritable "part de marché » pour des identités religieuses vécues en totale déconnexion des organisations ou des groupes religieux de référence. Seuls $3 \%$ des très nombreuses personnes qui ne déclarent aucune appartenance confessionnelle se sentent néanmoins «religieuses». Il n'y a pas individualisation totale du croire. On se sent religieux en fonction d'un lien, même ténu, à une confession.

\section{L'univers des croyances : des recompositions en cours}

Abordons à présent l'univers des croyances religieuses. On peut regretter que l'enquête ISSP ne consacre qu'un nombre réduit d'items à cette mesure. Le questionnaire est largement sous-tendu par une problématique chrétienne. Toutes les grandes croyances du christianisme ne sont pourtant pas mesurées. Et dans une situation où l'on parle de retour des valeurs religieuses et de nouvelles croyances, il serait important d'avoir des indicateurs nombreux pour tester les «nouvelles voies spirituelles" que nos contemporains peuvent expérimenter. Malgré la rareté des indicateurs de croyances, il est possible de lire dans les résultats à la fois l'affaiblissement des croyances les plus liées à l'univers chrétien et des recompositions en cours. Quatre questions permettent de mesurer assez finement la croyance en Dieu.

Pouvez-vous indiquer laquelle de ces affirmations correspond le mieux à ce que vous croyez à propos de Dieu?

- Je ne crois pas en Dieu 18 
17 - Je ne sais pas s'il y a un Dieu et je ne crois pas qu'il existe un moyen de le savoir 17

18 - Je ne crois pas en un Dieu personnel, mais je crois en une sorte de Puissance supérieure 14

19 - Je m'aperçois que je crois en Dieu à certains moments mais pas à d'autres 10

20 - Même si j'ai des doutes, j'ai l'impression que je crois en Dieu 19

21 - Je crois que Dieu existe réellement et je n'ai pas de doute à ce sujet 21

22 - Non réponse 2

23 Qu'est-ce qui décrit le mieux vos croyances au sujet de Dieu?

24 - Je ne crois pas en Dieu actuellement et je n'y ai jamais cru 21

25 - Je ne crois pas en Dieu actuellement mais j'y ai cru antérieurement 17

26 - Je crois en Dieu actuellement mais je n'y ai pas toujours cru 9

27 - Je crois en Dieu actuellement et j'y ai toujours cru 35

28 - Non réponse 18

Êtes-vous d'accord ou pas d'accord avec les opinions suivantes :

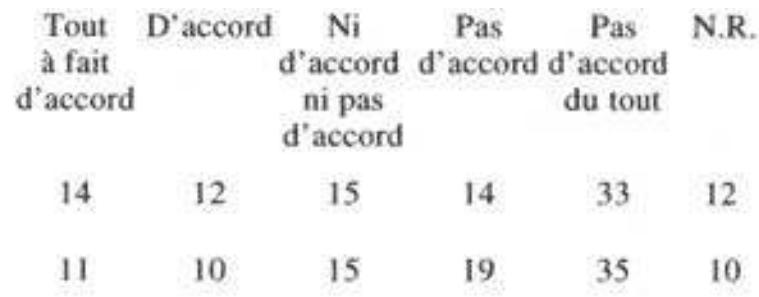

$\begin{array}{lllllll}\begin{array}{l}\text { Il existe un Dieu qui s'intéresse à } \\ \text { chaque être humain personnellement }\end{array} & 14 & 12 & 15 & 14 & 33 & 12 \\ \begin{array}{l}\text { Pour moi, la vie n'a de sens que si } \\ \text { Dieu existe }\end{array} & 11 & 10 & 15 & 19 & 35 & 10\end{array}$

29 Sur la première question, très détaillée, $35 \%$ ne croient pas en Dieu, $24 \%$ font des réponses ambiguës alors que $40 \%$ sont des croyants plus ou moins fermes. La deuxième question fait se situer les gens par rapport à leur passé. Les liaisons statistiques avec la question précédente montrent que cet indicateur fonctionne comme une mesure d'intensité de croyance. Autrement dit, les croyants actuels qui ne l'ont pas toujours été sont moins croyants que ceux qui y ont toujours cru, tandis que les incroyants actuels qui sont d'anciens croyants gardent davantage de doutes que les incroyants de toujours. Sur les deux dernières questions (croyance en un Dieu personnel qui s'intéresse à chacun et le sens de la vie donné par l'existence de Dieu), la croyance ferme est entre 21 et $26 \%$. Les différences dans les niveaux de croyance en Dieu selon les indicateurs montrent que la division des individus en seulement deux catégories, les croyants et les incroyants, représente une simplification. Il y a énormément de nuances dans les niveaux et dans les formes de la croyance en Dieu. 
Tableau 3 - Échelle de croyances en Dieu selon l'intégration au catholicisme (\% verticaux)

\begin{tabular}{|c|ccc|c|c|}
\hline & \multicolumn{3}{|c|}{ Catholique } & \\
\cline { 2 - 7 } & $\begin{array}{c}\text { Pratiquant } \\
\text { régulier } \\
(\mathrm{n}=108)\end{array}$ & $\begin{array}{c}\text { Pratiquant } \\
\text { irrégulier } \\
(\mathrm{n}=105)\end{array}$ & $\begin{array}{c}\text { Non } \\
\text { pratiquant } \\
(\mathrm{n}=195)\end{array}$ & $\begin{array}{c}\text { Sans religion } \\
(\mathrm{n}=367)\end{array}$ & Ensemble \\
\hline Nulle (échelle à 4) & 0 & 0 & 0 & 31 & 14 \\
Échelle 5-6 & 0 & 2 & 7 & 35 & 18 \\
Échelle 7-12 & 0 & 12 & 34 & 25 & 22 \\
Échelle 13-16 & 15 & 37 & 43 & 6 & 21 \\
Forte (Échelle 17 à 20) & 85 & 49 & 16 & 3 & 25 \\
\hline
\end{tabular}

Si les niveaux de croyance ne sont pas les mêmes selon l'indicateur retenu, ces questions sont cependant très fortement liées entre elles (avec des V de Cramer entre 0.44 et 0.56 ). Ceci autorise la création d'une échelle de croyance en Dieu $^{23}$. Cette échelle se révèle très fortement corrélée à l'intégration au catholicisme, comme le tableau 3 le montre $(\mathrm{V}$ de Cramer $=0.45^{24}$ ). Tous les pratiquants sont nettement des croyants. Par contre, à l'autre extrême, on trouve un petit pourcentage de sans religion qui ont pourtant quelques croyances, plus ou moins fermes, en une divinité ou en un Dieu. On a là des individus détachés des institutions religieuses mais qui n'en ont pas moins une certaine foi, probablement vécue sur un mode très original.

Tableau 4 - Croyances selon l'intégration au catholicisme

\begin{tabular}{|l|ccc|c|c|}
\hline \multirow{2}{*}{\begin{tabular}{l|c} 
Croire... \\
(certainement + probablement)
\end{tabular}} & $\begin{array}{c}\text { Catholigue } \\
\text { Pratiquant } \\
\text { régulier }\end{array}$ & $\begin{array}{c}\text { Pratiquant } \\
\text { irrégulier }\end{array}$ & $\begin{array}{c}\text { Non } \\
\text { pratiquant }\end{array}$ & $\begin{array}{c}\text { Sans } \\
\text { religion }\end{array}$ & Ensemble \\
\hline ... À la vie après la mort & 84 & 69 & 48 & 27 & 46 \\
...au ciel & 75 & 57 & 29 & 9 & 30 \\
...à l'enfer & 48 & 30 & 18 & 6 & 18 \\
...aux miracles religieux & 80 & 65 & 37 & 9 & 33 \\
\hline
\end{tabular}

Tableau 5 - Croyances selon l'échelle de croyances en Dieu

\begin{tabular}{|l|ccccc|c|}
\hline \multirow{2}{*}{$\begin{array}{c}\text { Croire... } \\
\text { (certainement + probablement) }\end{array}$} & \multicolumn{5}{|c|}{ Échelle de croyance en Dieu } & \\
\cline { 2 - 7 } & Forte $(17-20)$ & $13-16$ & $7-12$ & $5-6$ & Nulle (4) & Ensemble \\
\hline ... à la vie après la mort & 92 & 72 & 37 & 17 & 14 & 46 \\
$\ldots$... au ciel & 77 & 49 & 19 & 4 & 3 & 30 \\
$\ldots$ à l'enfer & 53 & 24 & 14 & 2 & 2 & 18 \\
... aux miracles religieux & 76 & 59 & 20 & 3 & 3 & 33 \\
\hline
\end{tabular}

D'autres croyances religieuses sont mesurées, croyance à la vie après la mort, au ciel, à l'enfer, aux miracles religieux (tableaux 4 et 5). L'adhésion à ces croyances est en fait très corrélée aux croyances en Dieu ( $\mathrm{V}$ de Cramer avec l'échelle entre 0.42 et 0.51 ) et à l'intégration au catholicisme. On est dans un univers de croyances communes dans le christianisme. Des différences apparaissent cependant dans les niveaux de croyance: l'enfer fait beaucoup moins recette que le ciel, les miracles religieux et surtout la vie après la mort. Le christianisme contemporain a opté plus que par le passé pour une vision optimiste de l'avenir et du salut. Les tableaux 4 et 5 montrent d'ailleurs que l'enfer est la seule croyance rejetée par une part importante de catholiques pratiquants et de croyants 
en l'existence de Dieu. L'autre phénomène notable sur ces tableaux concerne les sans religion et les gens refusant complètement l'existence d'un Dieu. Un pourcentage significatif de ces catégories accepte pourtant l'idée d'une vie après la mort. On a là un bel exemple de bricolage des croyances et d'une autonomie des individus qui peuvent rejeter les religions instituées et pourtant conserver ou recomposer un système de croyances religieuses.

Tableau 6 - Les croyances selon les âges

\begin{tabular}{|l|ccccc|c|}
\hline Croyance en... & $\begin{array}{c}18-24 \\
\text { ans }\end{array}$ & $\begin{array}{c}25-34 \\
\text { ans }\end{array}$ & $\begin{array}{c}35-49 \\
\text { ans }\end{array}$ & $\begin{array}{c}50-64 \\
\text { ans }\end{array}$ & $\begin{array}{c}65 \text { ans } \\
\text { et + }\end{array}$ & Ensemble \\
\hline ... la vie après la mort & 55 & 46 & 45 & 42 & 48 & 46 \\
... le ciel & 28 & 25 & 27 & 29 & 39 & 30 \\
$\ldots$. I'enfer & 22 & 15 & 16 & 17 & 22 & 18 \\
... les miracles religieux & 25 & 27 & 33 & 36 & 41 & 33 \\
\hline Échelle croyance Dieu fort (17-20) & 12 & 13 & 19 & 30 & 50 & 25 \\
\hline
\end{tabular}

Les jeunes générations étant celles où les recompositions de croyances ont le plus de chances d'apparaître, il vaut donc la peine de regarder la distribution de ces croyances par âge (tableau 6). Il apparait que la forte croyance en Dieu est rare chez les jeunes et nettement plus fréquente chez les vieux. Le même type de distribution, conforme à une hypothèse de sécularisation progressive du monde moderne, s'observe en partie pour la croyance aux miracles religieux. Par contre les différences selon les âges sont faibles pour les autres croyances et ne vont pas toujours dans le sens attendu. C'est particulièrement net pour la vie après la mort : cette croyance est non seulement à peu près aussi répandue dans toutes les classes d'âge mais ce sont même les 18-24 ans qui semblent y être les plus réceptifs. Leur niveau plus élevé de croyance vient de l'item « oui, probablement » et non pas du «oui, certainement». Sans adhérer à une foi religieuse particulière, sans nécessairement croire en Dieu, un certain nombre de jeunes veulent croire que la vie terrestre a un prolongement. Le même type de distribution était déjà repérable sur des données de la fin des années $1980^{25}$, montrant la spécificité de cette croyance et son découplage en cours par rapport à l'univers chrétien ${ }^{26}$.

Tableau 7 - Croyance en la vie après la mort (certaine + probable) selon l'intégration au catholicisme et l'âge

\begin{tabular}{|l|ccc|c|c|}
\hline & \multicolumn{3}{|c|}{ Catholique } & \multirow{2}{*}{ Sans religion } & Ensemble \\
\cline { 2 - 4 } & $\begin{array}{c}\text { Pratiquant } \\
\text { régulier }\end{array}$ & $\begin{array}{c}\text { Pratiquant } \\
\text { irrégulier }\end{array}$ & $\begin{array}{c}\text { Non } \\
\text { pratiquant }\end{array}$ & En & \\
\hline $18-34$ ans & 82 & 72 & 58 & 38 & 49 \\
$35-49$ ans & 100 & 76 & 50 & 25 & 45 \\
55 ans et plus & 81 & 56 & 35 & 11 & 45 \\
\hline Ensemble & 84 & 67 & 48 & 27 & 46 \\
\hline
\end{tabular}

Lecture du tableau : si en moyenne $46 \%$ de l'échantillon croient que la vie après la mort est certaine ou probable, $82 \%$ des catholiques pratiquants de 18-34 ans sont dans le même cas.

La particularité de la croyance à la vie après la mort apparaît encore plus clairement lorsqu'on prend en compte conjointement l'intégration au catholicisme et les âges, 
comme sur le tableau 7. Ce n'est que chez les pratiquants réguliers que la croyance en la vie après la mort est insensible à l'âge, cette croyance étant comme impliquée par le système théologique du catholicisme. Le plus étonnant est que chez les jeunes pratiquants irréguliers, non pratiquants et même sans religion, cette croyance tend à se maintenir, au point qu'elle est sensiblement plus fréquente chez les jeunes que chez les vieux. Près de $40 \%$ des jeunes qui se déclarent sans religion disent cependant croire à une vie après la mort (contre seulement $11 \%$ des sans religion de 55 ans et plus). Chez les jeunes, le découplage entre reconnaissance d'une appartenance religieuse institutionnelle et affirmation de certaines croyances religieuses est très net. On peut penser que certains jeunes déchristianisés, devenus insensibles à la croyance en Dieu et à l'idée de résurrection, découvrent parfois par le biais de l'ouverture aux autres cultures et traditions religieuses, l'espoir d'un futur au-delà de la vie. Cet espoir reste en général flou, plus probable que certain, il n'est pas associé à une représentation systématique du monde extrahumain.

Tableau 8 - Perception de la Bible selon l'intégration au catholicisme (\% verticaux)

\begin{tabular}{|l|cccc|c|c|}
\hline & \multicolumn{3}{|c|}{ Catholique } & \\
\cline { 2 - 5 } La Bible... & $\begin{array}{c}\text { Pratiquant } \\
\text { régulier }\end{array}$ & $\begin{array}{c}\text { Pratiquant } \\
\text { irrégulier }\end{array}$ & $\begin{array}{c}\text { Non } \\
\text { pratiquant }\end{array}$ & $\begin{array}{c}\text { Sans } \\
\text { religion }\end{array}$ & Ensemble \\
\hline ... parole même de Dieu & 18 & 9 & 3 & 1 & 5 \\
$\ldots$ inspirée par Dieu & 61 & 68 & 40 & 9 & 32 \\
... livre de I'histoire humaine & 7 & 16 & 37 & 64 & 43 \\
Cela ne me concerne pas & 1 & 1 & 7 & 18 & 11 \\
\hline
\end{tabular}

Une question était aussi consacrée au sens donné à la Bible. Question d'inspiration protestante mais fort intéressante justement à poser dans un pays de culture catholique, d'autant que le catholicisme tend à redécouvrir la Bible depuis quelques décennies. Il n'y a eu en France, à ma connaissance, qu'une enquête quantitative sur le sens de la Bible, réalisée en $1991^{27}$. $37 \%$ disaient posséder une Bible à leur domicile et $28 \%$ qu'il leur arrivait de la lire au moins occasionnellement (taux de lecture régulière : $5 \%{ }^{28}$ ). Le taux de lecture au moins occasionnelle montait à $58 \%$ chez les catholiques pratiquants réguliers et était quand même de $11 \%$ chez les sans religion, montrant déjà qu'une petite partie des sans religion pouvait s'intéresser aux textes sacrés. L'enquête ISSP ne mesure hélas pas les comportements de lecture, mais elle interroge sur la perception de la Bible. $5 \%$ des Français absolutisent la Bible et en ont une vision qu'on peut qualifier de fondamentaliste : « La Bible est la parole même de Dieu et elle doit être prise au pied de la lettre ». $32 \%$ en ont une perception religieuse mais plus ouverte : « La Bible représente la parole de Dieu mais elle ne doit pas être comprise à la lettre ». $43 \%$ la considèrent comme une œuvre humaine, témoignage de l'histoire : « La Bible est un livre ancien de contes, de légendes, d'histoire et de préceptes moraux rapportés par les hommes». Enfin $11 \%$ choisissent l'item "Tout cela ne me concerne pas ", probablement parce que pour eux, seules des personnes religieuses peuvent se prononcer sur le sens de la Bible et $10 \%$ ne répondent pas. Le tableau 8 confirme les résultats de l'enquête antérieure. Si la Bible est perçue comme un texte divin par la grande majorité des catholiques pratiquants, elle est au contraire vue comme purement humaine par la plupart des personnes qui rejettent les religions. Il n'en reste pas moins vrai qu'un petit public de sans religion (environ $10 \%$ des sans religion, soit $4.5 \%$ des Français) considère la Bible comme étant d'origine divine. 


\section{L'univers des croyances parallèles}

Outre les croyances relevant de l'univers du christianisme, présentées ci-dessus, l'enquête comporte une batterie de quatre questions optionnelles, qui n'ont donc pas été posées dans tous les pays mais seulement dans certains d'entre eux, dont la France. Cette batterie porte sur des croyances parallèles, souvent combattues par les grandes religions. S'agit-il vraiment de croyances religieuses? On pourrait bien sûr en débattre sociologiquement très longuement en fonction de la définition que l'on retient des religions. Ces croyances aux porte-bonheur, aux voyantes, aux guérisseurs, aux signes astrologiques n'impliquent pas nécessairement de croire en Dieu, ni même nécessairement à un monde surnaturel. Il s'agit de croyances autour du futur qu'il serait possible de prévoir et d'influencer grâce à des médiations plus ou moins puissantes et exceptionnelles. Nous qualifions ces croyances de parallèles dans la mesure où elles entretiennent des liens avec les croyances religieuses, tout en ne relevant pas exactement du même univers de sens ${ }^{29}$. Les résultats sont les suivants :

Pourriez-vous entourer une case sur chaque ligne ci-dessous selon que chaque affirmation est à votre avis vraie ou fausse?

- Les porte-bonheur apportent parfois

la chance

- Certaines voyantes peuvent vraiment

prévoir le futur

- Certains guérisseurs ont des pouvoirs donnés par Dieu pour guérir

- Le signe astrologique d'une personne

à la naissance ou l'horoscope peuvent

influer sur la vie future
Certaine- Probable- Probable- Certaine- N.R.
ment vrai ment vrai ment faux ment faux

$\begin{array}{lllll}2 & 22 & 23 & 47 & 7 \\ 8 & 29 & 23 & 33 & 7 \\ 10 & 28 & 20 & 33 & 8 \\ 7 & 33 & 22 & 30 & 9\end{array}$

Peu de personnes croient fermement aux porte-bonheur, aux voyantes, aux guérisseurs inspirés par Dieu, au signe astrologique et à l'horoscope. Mais si l'on ajoute les rares croyants fermes et les nombreux moins convaincus (total des certainement et des probablement vrai), on atteint des pourcentages qui ne sont pas très différents des grandes croyances du christianisme. Les enquêtes menées en France depuis le début des années 1980 montrent d'ailleurs une certaine progression dans les niveaux de croyance. Ces quatre variables sont fortement liées entre elles, quoiqu'un peu moins fortement que les précédentes croyances ( $\mathrm{V}$ de Cramer à $0.38,0.38,0.36,0.30,0.30,0.23)$. On peut construire une échelle en dénombrant pour chaque individu son nombre de croyances parallèles déclarées ${ }^{30}$. Les adeptes de cet univers «hétérodoxe " constituent $24 \%$ de l'échantillon, les indécis sont $39 \%$ et ceux qui le rejettent $37 \%$. Signalons quelques traits saillants de la population croyante. Elle est à majorité féminine (la surreprésentation des femmes est plus forte pour ces croyances que pour la croyance en Dieu ou l'intégration au catholicisme ${ }^{31}$ ). Alors que la croyance en Dieu est comme on l'a vu nettement liée à l'âge, l'échelle de croyances parallèles l'est peu ${ }^{32}$. Ce sont les personnes âgées qui sont les plus résistantes au phénomène, probablement parce que leur culture religieuse est fortement structurée par l'univers du christianisme, même lorsqu'elles ont pris leurs distances avec le catholicisme. Elles ont probablement aussi été très marquées par la critique rationaliste des croyances «populaires». Même si les personnes âgées ont en moyenne un bagage 
scolaire faible, elles savent qu'aux yeux de la science, les croyances "populaires » sont illégitimes.

La tradition d'études a montré qu'on pouvait en fait distinguer deux catégories, les croyances aux phénomènes paranormaux qui peuvent revendiquer une certaine légitimité scientifique (comme la transmission de pensée, les rêves prémonitoires, les extra-terrestres) et les croyances qui ont trait à la prévision du futur. Les premières sont plus fréquentes parmi les cadres et les personnes fortement diplômées alors que les secondes concernent prioritairement le bas des classes moyennes, à petit niveau de diplôme. Nos quatre indicateurs relèvent essentiellement de la seconde catégorie, la maîtrise de son futur par des médiations " extraordinaires ». Nos données confirment les analyses antérieures. Ainsi l'adhésion aux croyances parallèles passe de $14 \%$ chez les cadres supérieurs à $29 \%$ chez les professions intermédiaires, $30 \%$ chez les employés et ouvriers, $32 \%$ chez les artisans et commerçants. Les personnes qui se perçoivent comme appartenant aux classes supérieures sont très peu croyantes, le maximum étant au contraire atteint chez ceux qui se perçoivent comme appartenant à la classe ouvrière (33\%). Selon les niveaux d'éducation, les plus croyants sont ceux qui ont fait des études de durée moyenne ou courte, alors que les incrédules ont plus souvent fait de longues études (au-delà de 20 ans). Mais au total, ces relations ne sont que d'intensité moyenne.

Les liens entre l'univers des croyances parallèles et celui de la religion sont en fait complexes. Ainsi, les relations entre le sentiment d'être religieux et l'échelle d'adhésion aux croyances parallèles ne sont pas très étroits $(\mathrm{V}$ de Cramer $=0.17)$. Ceux qui se disent non religieux rejettent souvent ces croyances, probablement au même titre qu'ils rejettent les croyances proprement religieuses, perçues comme inutiles ou nuisibles. Seulement $35 \%$ des croyants parallèles se disent religieux (alors que c'est le cas de $51 \%$ des catholiques et de $89 \%$ des pratiquants). Ils semblent hésiter à voir dans leur foi un véritable univers religieux. La condamnation des religions traditionnelles à l'égard de ces pratiques pourrait expliquer l'auto-distanciation des croyants parallèles à l'égard d'un sentiment d'identité religieuse. Les religions instituées ont de fait monopolisé la définition sociale d'une religion. Ceux qui croient en un religieux non orthodoxe ont donc $\mathrm{du}$ mal à se sentir religieux car ils ne partagent pas l'univers des croyances et des pratiques conformes.

Tableau 9 - Échelle de croyances parallèles selon l'intégration au catholicisme (\% verticaux)

\begin{tabular}{|l|ccc|c|c|}
\hline & \multicolumn{3}{|c|}{ Catholique } & \multirow{2}{*}{} \\
\cline { 2 - 5 } & $\begin{array}{c}\text { Pratiquant } \\
\text { régulier }\end{array}$ & $\begin{array}{c}\text { Pratiquant } \\
\text { irrégulier }\end{array}$ & $\begin{array}{c}\text { Non } \\
\text { pratiquant }\end{array}$ & $\begin{array}{c}\text { Sans } \\
\text { religion }\end{array}$ & Ensemble \\
\hline - Rejet & 41 & 25 & 25 & 49 & 37 \\
- Indécision & 47 & 42 & 42 & 33 & 39 \\
- Croyance & 12 & 32 & 34 & 18 & 24 \\
\hline
\end{tabular}

Cette complexité des relations entre croyances parallèles et religion dominante est également visible sur le tableau $9(\mathrm{~V}=0.20)$. Les sans religion rejettent plus souvent ce type de croyance. Les plus « croyants parallèles " sont les catholiques non pratiquants, donc ceux qui, du point de vue de l'intégration au catholicisme, sont en position intermédiaire, de liens mous avec leur religion de référence. La relation n'est pas très intense et, de fait, toutes les catégories de catholiques sont en partie touchées par ce type 
de croyances, y compris les pratiquants réguliers. On retrouve ici ce que les enquêtes précédemment citées ont mis en lumière.

41 Les études antérieures semblaient aussi montrer que les croyances parallèles croissaient linéairement avec la croyance en Dieu et surtout avec la croyance en la vie après la mort et aux miracles ${ }^{33}$. Comme si l'on avait là un univers de recomposition de croyances en un surnaturel souvent déconnecté de l'univers du christianisme. Les résultats de l'enquête ISSP sont voisins sans être identiques. D'après le tableau 10 (V de Cramer $=0.25)$, les croyances parallèles sont les plus développées chez ceux dont la croyance en Dieu est relativement forte sans être la plus élevée. De la même manière, la liaison entre croyances parallèles, vie après la mort et miracles n'est pas complètement linéaire (tableau 11 avec respectivement $\mathrm{V}=0.26$ et 0.27 ). Si l'on exclut les incroyants qui n'acceptent pas plus Dieu, l'après mort et les miracles que les «superstitions » sur le futur, toutes les autres catégories sont touchées par les recompositions de croyances. Mais ce ne sont pas les croyants les plus intégrés à l'univers chrétien qui sont les plus marqués par la croyance en un «surnaturel hétérodoxe». Les croyances orthodoxes continuent pour les plus intégrés au catholicisme à faire système et à freiner leur adhésion à un univers hétérodoxe.

Tableau 10 - Échelle de croyances populaires selon l'échelle de croyance en Dieu (\% verticaux)

\begin{tabular}{|l|cccccc|c|}
\hline & \multicolumn{5}{|c|}{ Échelle de croyance en Dieu } & \\
\cline { 2 - 6 } & Forte (17-20) & $13-16$ & $7-12$ & $5-6$ & Nulle (4) & Ensemble \\
\hline - Rejet & 33 & 20 & 34 & 56 & 68 & 37 \\
- Indécision & 42 & 42 & 39 & 32 & 27 & 39 \\
- Croyance & 25 & 38 & 27 & 12 & 5 & 24 \\
\hline
\end{tabular}

Tableau 11 - Pourcentage de « croyants parallèles » selon la croyance à la vie après la mort et aux miracles

\begin{tabular}{|l|cccc|c|}
\hline & \multicolumn{2}{|c|}{$\begin{array}{c}\text { Oui, } \\
\text { certainement probablement probablement certainement } \\
\text { Croire }\end{array}$} & \multicolumn{7}{|c|}{$\begin{array}{c}\text { Oui, } \\
\text { pas }\end{array}$} & pas & \\
\hline - à la vie après mort & 33 & 35 & 16 & 11 & 24 \\
- aux miracles religieux & 37 & 37 & 27 & 10 & 24 \\
\hline
\end{tabular}

Lecture du tableau: si en moyenne $24 \%$ de l'échantillon sont réceptifs aux croyances parallèles, $33 \%$ de ceux qui croient de façon certaine à la vie après la mort et $35 \%$ de ceux qui y croient probablement sont aussi des croyants parallèles.

\section{Identités religieuses et systèmes de valeurs}

Image mitigée des organisations religieuses, détachement progressif du catholicisme, recomposition des systèmes de croyances, telles semblent être quelques grandes tendances du panorama religieux de la France contemporaine. Il nous reste à nous poser la question de l'effet des identités religieuses sur l'ensemble des attitudes. L'impact des religions sur une société se mesure en effet non seulement par l'extension des pratiques et des croyances, mais aussi par l'impact que les choix religieux ont sur les systèmes de valeurs. Nous allons voir que la construction d'une identité religieuse à travers une identification à un groupe, la participation à ses activités, l'adhésion à un système de 
croyances ou à l'inverse la construction d'une identité a-religieuse produit des effets sur l'ensemble du système de valeurs des individus. Pour le montrer, nous chercherons quels sont les liens entre toute une série d'attitudes et l'intégration au catholicisme, puisque cette dimension semble toujours importante. Par rapport aux tableaux précédents, nous avons distingué deux catégories de sans religion. Dans la mesure où ils sont très nombreux et où on a pu déceler l'existence d'un groupe de sans religion qui avaient pourtant certaines croyances religieuses, nous distinguerons des sans religion croyants et des sans religion non croyants $^{34}$. L'objectif est de voir si cette distinction a des effets en termes de systèmes d'attitudes.

Le tableau 12 présente les résultats de trois échelles, une sur la légitimité des relations sexuelles en dehors du couple légal ${ }^{35}$, une sur la conception des rôles à l'intérieur du couple et de la famille ${ }^{36}$, une sur la légitimité du recours à l'avortement ${ }^{37}$. L'opinion est globalement très ouverte en matière de liberté sexuelle (sauf pour les relations extraconjugales et pour les relations homosexuelles), de conception des rôles parentaux et de liberté de l'avortement. Mais la distribution des opinions est très différente selon l'intégration au catholicisme ( $\mathrm{V}$ de Cramer respectivement de $0.41,0.22$ et 0.37$)^{38}$. Lorsqu'on contrôle l'effet de l'âge, on vérifie que si les jeunes sont en moyenne plus tolérants que les personnes âgées, pour chaque classe d'âge, un fort effet d'intégration au catholicisme subsiste. Sur ces attitudes morales et familiales, l'identité religieuse a toujours des effets considérables. Le fait d'adhérer à quelques croyances pour les sans religion n'a par contre pas de conséquence très claire sur ces attitudes.

Tableau 12 - Les valeurs sexuelles et familiales selon l'intégration au catholicisme

\begin{tabular}{|c|c|c|c|c|c|c|}
\hline & \multicolumn{3}{|c|}{ Catholique } & \multicolumn{2}{|c|}{ Sans religion } & \multirow[b]{2}{*}{ Ensemble } \\
\hline & $\begin{array}{l}\text { Pratiquant } \\
\text { régulier }\end{array}$ & $\begin{array}{l}\text { Pratiquant } \\
\text { irrégulier }\end{array}$ & $\begin{array}{c}\text { Non } \\
\text { pratiquant }\end{array}$ & Croyant & $\begin{array}{c}\text { Non } \\
\text { croyant }\end{array}$ & \\
\hline - Relations sexuelles toujours légitimes & 9 & 38 & 52 & 79 & 67 & 53 \\
\hline - Rôle moderne hornme et femme & 26 & 42 & 53 & 65 & 63 & 52 \\
\hline - Avortement toujours légitime & 38 & 68 & 79 & 78 & 89 & 75 \\
\hline
\end{tabular}

Lecture du tableau : si en moyenne $53 \%$ de l'échantillon légitiment les relations sexuelles en dehors du couple légal, ce n'est le cas que de $9 \%$ des catholiques pratiquants réguliers.

D'autres valeurs sont dépendantes de l'intégration au catholicisme, mais pas toujours de façon très intense (tableau $13^{39}$ ). Ainsi, $54 \%$ estiment qu'il est illégitime de tricher dans sa déclaration d'impôts pour payer moins d'impôts et de faire des fausses déclarations pour obtenir des pouvoirs publics des allocations indues ${ }^{40}$. Mais les catholiques bien intégrés à leur religion ne sont que modérément plus moraux que les autres sur cette dimension, les sans religion étant les plus tolérants pour ce type d'incivilité. L'item suivant correspond à une situation particulière d'accident dans lequel un piéton est heurté par une voiture en excès de vitesse. Les interviewés doivent dire s'il est légitime de faire un faux témoignage pour innocenter le chauffeur qui se trouve être un ami. À nouveau, beaucoup de Français rejettent la légitimité du faux témoignage au nom de l'amitié. Les catholiques ne sont que légèrement plus intransigeants que les sans religion. 
Tableau 13 - Les valeurs morales et politiques selon l'intégration au catholicisme

\begin{tabular}{|c|c|c|c|c|c|c|}
\hline \multirow[t]{2}{*}{$=$} & \multicolumn{3}{|c|}{ Catholique } & \multicolumn{2}{|c|}{ Sans religion } & \multirow[b]{2}{*}{ Ensemble } \\
\hline & $\begin{array}{l}\text { Pratiquant } \\
\text { régulier }\end{array}$ & $\begin{array}{l}\text { Pratiquant } \\
\text { irrégulier }\end{array}$ & $\begin{array}{c}\text { Non } \\
\text { pratiquant }\end{array}$ & Croyant & $\begin{array}{l}\text { Non } \\
\text { croyant }\end{array}$ & \\
\hline $\begin{array}{l}\text { - Mal de tricher sur les impôts et les } \\
\text { allocations }\end{array}$ & 71 & 60 & 52 & 46 & 51 & 54 \\
\hline $\begin{array}{l}\text { - Pas légitime de faire un faux témoignage } \\
\text { pour défendre un ami }\end{array}$ & 72 & 68 & 65 & $\$ 3$ & 61 & 63 \\
\hline - La nature humaine est bonne & 45 & 46 & 37 & 33 & 36 & 39 \\
\hline - Perception négative de la science & 58 & 32 & 17 & 23 & 16 & 25 \\
\hline - Favorable à la peine de mort & 32 & 32 & 50 & 36 & 35 & 38 \\
\hline $\begin{array}{l}\text { - La laicité a l'ecole, assouplie ou } \\
\text { abandonnée }\end{array}$ & 46 & 18 & 15 & 18 & 6 & 17 \\
\hline - Se positionne à gauche (1-4 sur l'échefle) & 14 & 23 & 26 & 41 & 59 & 36 \\
\hline - Partis de la gauche plurielle & 18 & 26 & 30 & 44 & 60 & 39 \\
\hline - Partis de droite et d'extrême droite & 58 & 41 & 31 & 20 & 12 & 28 \\
\hline
\end{tabular}
conception de la nature humaine et de la science. L'optimisme à l'égard de la nature humaine ${ }^{41}$ est un peu plus développé chez les catholiques pratiquants que chez les sans religion. Mais là encore, les écarts sont faibles. La perception de la science est mesurée par deux items ${ }^{42}$ agglomérés en échelle. Ici les écarts en fonction de l'intégration religieuse sont très importants. Les sans religion semblent assez inconditionnels de la science, ce qui n'est pas le cas des catholiques pratiquants. Mais l'intensité de cette relation est largement due au second item qui compare la science et la foi. Sur le premier, les différences selon l'intégration au catholicisme existent mais elles sont limitées.

La dernière partie du tableau porte sur des choix politiques et d'abord l'acceptation ou le rejet de la peine de mort ${ }^{43}$. Les écarts ne sont pas très grands mais le plus remarquable est ici la forme de la distribution : ce sont les catholiques non pratiquants qui soutiennent le plus la mise en œuvre de la peine de mort. Alors que les pratiquants et les sans religion sont plus réservés. La doctrine catholique, très réservée à l'égard de la peine de mort même si elle n'en condamne pas absolument le principe, semble avoir ici un impact sur les prises de position des individus, au point qu'ils sont même légèrement moins favorables à la peine capitale que les sans religion, qui ont en principe davantage intégré les valeurs de la modernité et les valeurs de gauche (cf. ci-après). Une question spécifique à la France évoque la laïcité à l'école. Cette question est très sensible puisqu'elle touche au statut de l'institution catholique dans la société française. Si, dans l'ensemble de l'échantillon, la réponse majoritaire est pour le statu quo et si une minorité non négligeable veut le renforcement de cette laïcité, peu nombreux sont pour son abandon ou même son assouplissement. Les réponses sont très marquées par l'identité religieuse des individus et par leurs référents communautaires. Ce sont bien sûr les sans religion qui souhaitent très souvent le renforcement alors que l'abandon ou l'assouplissement sont surtout demandés par les catholiques pratiquants réguliers ${ }^{44}$. L'avant-dernier item présenté, l'échelle gauche-droite en 10 positions, dont les quatre positions de gauche sont ici retenues, montre aussi, comme on pouvait s'y attendre par des enquêtes précédentes, une forte relation avec l'intégration au catholicisme. Les sans religion ${ }^{45}$ sont très nettement orientés à gauche alors que les catholiques pratiquants sont souvent à droite. Cette tradition séculaire continue de se maintenir même s'il est possible, d'après certaines enquêtes, que la force de la relation faiblisse un peu $^{46}$. Quant au dernier 
indicateur, la proximité partisane, il confirme les liaisons observées sur le précédent. Les sans religion, surtout non croyants, sont très souvent sympathisants des partis de gauche alors que les catholiques pratiquants réguliers expriment très souvent leur accord avec les partis de droite ${ }^{47}$.

Le degré d'intégration au catholicisme continue d'introduire des différences fortes dans les systèmes d'attitudes des Français. Il convient d'analyser aussi si l'adhésion au système de croyances parallèles introduit le même type de distinctions. La réponse est négative. Lorsqu'on croise les dimensions prises en compte dans les tableaux 12 et 13 avec l'échelle de croyances parallèles, on obtient une absence de relations pour les valeurs sexuelles et morales, des relations faibles pour les valeurs politiques (tableau 14). Ces corrélations sont liées à l'attitude de rejet. Ceux qui refusent les croyances parallèles (qui ont aussi tendance à rejeter les croyances orthodoxes au nom d'un attitude rationaliste) sont plutôt orientés à gauche alors que les indécis et les croyants sont un peu plus souvent à droite (ou à distance de la politique). L'univers des croyances parallèles ne constitue donc pas un clivage fort comparable à celui qu'a produit le catholicisme. Les systèmes d'attitudes des Français sont largement construits autour de l'univers chrétien ou sur l'affirmation de l'irréligion.

Tableau 14 - Les valeurs politiques selon l'échelle de croyances parallèles

\begin{tabular}{|l|ccc|c|}
\hline & Croyance & Indécision & Rejet & Ensemble \\
\hline - Favorable à la peine de mort & 48 & 42 & 29 & 38 \\
- Se positione à gauche (1-4 sur l'échelle) & 28 & 31 & 45 & 36 \\
\hline - Partis de la gauche plurielle & 31 & 35 & 45 & 39 \\
- Partis de droito et d'cxtrême droite & 29 & 29 & 28 & 28 \\
\hline
\end{tabular}

\section{Conclusion}

Au total, nous n'avons pas trouve dans cette enquête de grands bouleversements par rapport aux tendances antérieures. Le processus de sécularisation et d'affaiblissement du catholicisme se poursuit, la religion dominante n'est pas remplacée par d'autres systèmes religieux forts et homogènes. Il est donc difficile de parler d'un vrai retour du religieux. On a l'impression que le système catholique perd de son homogénéité, qu'il est vécu sur un mode plus individualisé et que ceux qui s'en détachent peuvent cependant maintenir ou recomposer des éléments de croyances religieuses. Le religieux est moins régulé par le catholicisme, il est plus diffus et disséminé, il est plus composite et hétérodoxe. Les sans religion eux-mêmes semblent moins homogènes, hésitant entre 1'affirmation de la rationalité et de la science et 1'adhésion à la croyance en la vie après la mort ou à certaines croyances parallèles. Ils se définissent moins nettement en opposition aux systèmes religieux. Il y a individualisation des références religieuses, les grands systèmes de sens sont moins qu'autrefois des prêts à porter, ce ne sont que des repères dans lesquels chacun puise assez librement. L'attitude religieuse continue d'être une dimension importante de la construction de l'identité des individus, tout leur système de pensée est marqué par la position qu'ils adoptent en matière religieuse, mais ces identités sont de plus en plus complexes, nuancées, souvent vécues sur le mode du possible et non pas des grandes certitudes. Bien sûr, les Français sont de moins en moins nombreux à avoir une identité fortement structurée par le catholicisme. Ils ont plutôt une identité 
structurée par I'irréligion. Cette montée de I'irréligion explique probablement une grande partie de l'évolution des valeurs vers une grande tolérance pour les écarts aux normes morales et sexuelles, vers un rejet des normes autoritaires et des valeurs traditionnelles.

\section{NOTES}

1. Je remercie Roland Campiche et Yves Lambert pour leurs stimulantes réactions à une première version de ce texte, présenté dans une session thématique de la $\mathrm{XXV}^{\mathrm{e}}$ conférence de la Société internationale de sociologie des religions, Leuven, juillet 1999.

2. L'ISSP réalise chaque année une enquête avec un questionnaire identique dans plus de 25 pays. Chaque module annuel dure 15 minutes (sans compter les variables socio-démographiques) et est consacré à un thème spécifique, qui a vocation à être répliqué au bout de quelques années.

3. Une équipe d'enseignants et de chercheurs s'est constituée en un groupe ISSP-France qui réunit Yannick Le mel (INSEE, CREST-LSQ), Louis Chauvel et Olivier Galland (FNSP, OSC), Alain Degenne et Michel Forsé (CNRS, LASMAS), Bruno Cautrès et moi-même (CNRS, CIDSP). Yves Lambert (CNRS, GSRL) a été associé à la réalisation du module religion en 1998. Nous ne bénéficions pour cette opération que de tous petits moyens alloués par le CNRS, l'INSEE, la FNSP et nos différents laboratoires. Le questionnaire est envoyé par poste à des personnes tirées au hasard sur la liste téléphonique nationale. La personne du ménage qui doit répondre est définie selon une méthode des anniversaires. L'enquête sur la religion, administrée à la fin de 1998, comporte 1133 réponses valides. Les résultats présentés ici ont été pondérés pour rétablir une bonne représentativité de l'échantillon. Les données des enquêtes ISSP, en France et dans le monde, peuvent être obtenues auprès du Centre d'Informatisation des données socio-politiques, Banque de données socio-politiques, IEP de Grenoble, BP 48, 38040 Grenoble cedex 9.

4. L'enquête Valeurs a été renouvelée au printemps 1999. Nous n'utiliserons pas ici les résultats récents qui seront analysés dans un ouvrage collectif: BRÉCHON Pierre (éd.), Les valeurs des Français : évolutions de 1981 à 1999, Armand Colin, à paraître, automne 2000.

5. Une question de l'enquête Valeurs de 1981 donnait des résultats voisins. $14 \%$ disaient qu'il existe « une seule vraie religion », $52 \%$ que « dans toutes les grandes religions du monde, on peut trouver des vérités et des significations fondamentales » et $26 \%$ que «aucune n'a de vérité à offrir ». La principale différence réside dans l'affaiblissement de la " religion exclusiviste ».

6. Sondage SOFRES pour le Figaro-Magazine (22-24 novembre 1994).

7. Sondage SOFRES pour Actuel (10-14 janvier 1992).

8. Sondage SOFRES pour 7 sur 7 (septembre-octobre 1994).

9. Sondage SOFRES pour L'Express (21-23 novembre 1989).

10. Sondage SOFRES pour 7 sur 7 (28-30 août 1996)

11. Cf. Hugues PORTELLI, «L'évolution politique des catholiques », dans SOFRES, L'état de l'opinion, 1994, Seuil, pp. 179-199. Le phénomène est confirmé par l'enquête ISSP : une action proprement politique des Églises est rejetée par une majorité d'enquêtés.

12. Ils sont jugés très utiles par $38 \%$ des Français et plutôt utiles par $46 \%$. Sondage SOFRES pour le Pèlerin-Magazine (30 septembre-5 octobre 1994). 
13. On trouve peu de catholiques pratiquants réguliers approuvant les prises de position du pape et des évêques sur la morale sexuelle. Et la moitié d'entre eux trouvent injustifiée la sanction prise par le pape contre Mgr Gaillot (SOFRES pour 7 sur 7, janvier-février 1995).

14. Cette individualisation de la culture catholique est bien mise en évidence dans les travaux de Jean-Marie donegani, La liberté de choisir. Pluralisme religieux et pluralisme politique dans le catholicisme français contemporain, Paris, Presses de la FNSP, 1993.

15. Cf. Jean-Paul willaime, "Religious and Secular France Between Northern and Southern Europe », Social Compass, 45/1, 1998, pp. 155-174.

16. Comme beaucoup d'enquêtes, celle-ci minore la place des musulmans, parce qu'ils répondent plus difficilement aux enquêtes par questionnaire. S'ils étaient justement représentés dans l'enquête, les autres religions devraient être autour de $10 \%$.

17. Ainsi le mouvement sectaire, s'il connaît un développement, reste si minoritaire qu'il n'apparaît pas dans les enquêtes statistiques. Sa place réelle dans la société française est sans commune mesure avec son importance dans les médias. Cf. Pierre BRÉCHON et Jean-Paul wiLLAIME (éds.), Médias et religions en miroir, PUF, (Coll. « Politique d'aujourd'hui »), à paraître (juin 2000).

18. Les items de la question "Tous les combien participez-vous à des activités religieuses ou d'organisations religieuses en dehors du culte?» sont ici recodés. L'échelle proposée allait de « jamais » à " plusieurs fois par semaine ». Par ailleurs, une autre question mesure l'intensité du bénévolat accompli depuis un an pour un groupe religieux. $15 \%$ des répondants ont fait au moins 1 ou 2 fois du bénévolat religieux pendant l'année d'implication. Un V de Cramer autour de 0.15 indique en général l'existence d'une relation peu intense mais statistiquement significative. Lorsque, dans la mesure des phénomènes d'opinion, on atteint des coefficients autour de 0.50 , c'est qu'on est en présence d'une relation très étroite.

19. Le V de Cramer est un indicateur statistique qui mesure l'intensité d'une corrélation. Il varie entre 0 et 1 . Un coefficient proche de 0 signale une absence de relation et une valeur proche de 1 une relation

20. Les items de la question « Tous les combien priez-vous environ? » sont ici recodés. L'échelle proposée allait de « jamais » à " plusieurs fois par jour ».

21. À la différence de l'enquête Valeurs et des Eurobaromètres, la mesure du sentiment religieux se fait ici en 7 positions, chacun devant s'identifier d'extrêmement religieux à extrêmement non religieux. 30 \% choisissent en fait le pôle religieux, 37 \% le pôle non religieux et 29 \% la position centrale (ni religieux, ni non religieux).

22. $89 \%$ des religieux se déclarent catholiques, $6 \%$ se classent en autres religions et $5 \%$ disent ne pas appartenir à une confession.

23. Elle est construite en ajoutant les scores obtenus à chaque question après avoir inversé les trois dernières et en éliminant le code 8 des sans réponse. Nous obtenons ainsi une échelle allant de 4 (individus qui sont toujours sur la réponse la plus incroyante) à 20 (individus qui choisissent toujours l'item le plus croyant). 803 individus sont classés sur cette échelle qui élimine les nonréponse.

24. Ceci confirme une fois de plus ce que Guy Michelat a bien démontré: l'intensité de l'assistance à la messe est un indicateur synthétique de mesure de l'intégration au catholicisme. À travers ce critère simple, c'est l'insertion dans l'univers catholique qui est mesuré. Cf. Guy MiChelat, "L'identité catholique des Français, 1. Les dimensions de la religiosité et 2. Appartenance et socialisation religieuse ", Revue française de sociologie, 31/3, juillet-sept. 1990, pp. 355-388 et 31/4, oct.-déc. 1990, pp. 609-633.

25. Cf. Guy michelat, "Pratiques et croyances religieuses : détachements et hétérodoxies ", in Yves lambert, Guy michelat (éds.), Crépuscule des religions chez les jeunes? Jeunes et religions en France, L'Harmattan, 1992, pp. 43-63 (coll. «Logiques sociales»); Yves LAMBERT, « Un paysage 
religieux en profonde mutation ", in Hélène RIFFAULT (éd.). Les valeurs des Français, Paris, PUF, 1994, pp. 123-162(coll. « Sociologies»).

26. La question sur la vie après la mort figure aussi dans les enquêtes Valeurs, pour lesquelles on disposera bientôt de trois points d'observation (1981, 1990, 1999). Elle y est posée sous une forme dichotomique, ce qui rend les comparaisons difficiles avec l'enquête ISSP. D'après les enquêtes Valeurs, les générations de l'après-guerre sont aujourd'hui un peu plus croyantes à la vie après la mort que les autres et cette croyance est devenue pour ces générations plus fréquente au fil du temps. Cf. Les valeurs des Français. Évolution de 1981 à 1999, à paraître.

27. Enquête initiée par la direction du développement de Bayard-Presse. Cf. Jean-François BARBIER-BOUVET, Les Français et la Bible, Bayard-Presse, Direction du développement, Bureau d'études, 1991.

28. Les motivations de lecture sont avant tout éthiques (trouver un guide moral et une aide pour l'action) mais elles sont aussi culturelles (mieux comprendre les racines de notre culture) et spirituelles (trouver des éléments pour la prière).

29. Cet univers de croyances a déjà été analysé en France depuis vingt ans, notamment par Frédéric Bon, Daniel Boy, Guy Michelat. Cf. notamment Daniel Boy et Guy MICHELAT, «Croyances aux para-sciences: dimensions sociales et culturelles", Revue française de sociologie, avril-juin 1986, pp. 175-204 ; Daniel Boy et Guy MICHELAT, « Les Français et les para-sciences », dans SOFRES, L'État de l'opinion 1994, pp. 201-217.

30. Pour chaque individu, l'échelle va de 0 (aucune croyance choisie) à 4 s'il croit au moins mollement aux quatre croyances. L'échelle a été recodée en 3 positions : rejet (aucune croyance admise), indécision ( 1 ou 2 items positifs), croyance ( 3 ou 4 items positifs).

31. La différence de genre concernant les attitudes religieuses est un phénomène très résistant dans le temps et observable dans de très nombreux pays. Cf. Pierre BRÉCHON, « Le mystère des identités religieuses masculines et féminines ", in Françoise LAUTMAN (éd.), Ni Ève ni Marie, Genève, Labor et Fides, 1997, pp.307-328; voir aussi le numéro thématique des Archives de Sciences Sociales des Religions, juillet-septembre 1996, $\mathrm{n}^{\circ} 95$.

32. Les $25-34$ ans partagent un peu plus ces croyances que les 18-24 ans. D'après une enquête française de 1989 et une enquête dans l'agglomération grenobloise en 1990, les croyances au paranormal et à l'astrologie étaient plus fréquentes chez les jeunes, ce qui n'est pas aussi net ici. Cf. Guy michelat, Pratiques et croyances religieuses, op. cit., p.56 et Jean-Paul BOzONNET, Pierre BRÉCHON, Jacques DERVILLE, Gilles IVALDI, Rapport de sondage 1990 - agglomération grenobloise, Grenoble, IEP de Grenoble, pp. 83-108.

33. Cf. Daniel boy et Guy michelat, « Les Français et les para-sciences », op. cit.

34. Ces derniers ne sont jamais situés sur les deux échelons les plus croyants de l'échelle de croyance en Dieu et des quatre questions sur la vie après la mort, le ciel, l'enfer et les miracles religieux. Au contraire, les sans religion croyants sont au moins sur l'une des cinq dimensions positionnées sur les deux échelons les plus croyants. Selon ce critère, sur 489 sans religion, 326 sont non croyants et 163 croyants.

35. Échelle bâtie sur quatre items fortement liés entre eux (légitimité des relations sexuelles avant le mariage, des relations sexuelles entre deux adultes de même sexe, de la cohabitation sans intention de se marier, de la cohabitation avant un mariage). Nous n'avons pas retenu dans l'échelle la légitimité des relations extraconjugales, car l'item est moins fortement lié avec les quatre autres. Nous retenons ici tous ceux qui trouvent au moins en partie légitime chacun des quatre comportements retenus.

36. Échelle construite sur deux indicateurs : «Le rôle de l'homme est de gagner l'argent du ménage, celui de la femme de s'occuper de la maison et de la famille » et "Tout bien pesé, lorsque la femme travaille à temps plein, la vie de famille en souffre ». Son retenus ici tous ceux qui rejettent, au moins partiellement, ces deux opinions. 
37. Là encore, on retient ceux qui rejettent, au moins en partie, les deux indicateurs suivants : «Il est mal pour une femme d'avoir un avortement en cas de :

- risque sérieux de grave malformation de l'enfant

- si la famille a un très bas revenu et ne peut subvenir à un enfant de plus ».

38. Nous aurions pu tout aussi bien utiliser ici le sentiment d'être religieux ou l'échelle de croyance en Dieu que l'intégration au catholicisme mesurée par l'assistance à la messe. Les liaisons ont une intensité très voisine de celle qui est observée avec l'intégration au catholicisme. 39. Le contrôle de l'âge ne fait cependant pas disparaître l'effet de l'intégration au catholicisme, sauf peut-être pour la nature humaine (cas litigieux).

40. Ce résultat provient d'une échelle qui isole ceux qui disent qu'il est mal ou très mal d'avoir ces deux comportements.

41. Il s'agit d'une question optionnelle, qui n'a donc pas été posée dans tous les pays, et qui fait se positionner les individus sur une échelle en 7 positions, allant de : «la nature humaine est fondamentalement bonne" à "la nature humaine est fondamentalement mauvaise et corrompue ». Nous retenons ici le pôle optimiste correspondant aux notes 1-3.

42. Sur les deux indicateurs «Dans l'ensemble, la science moderne fait plus de mal que de bien » et « Nous faisons trop confiance à la science et pas assez à la foi religieuse ", chaque enquêté est invité à formuler sa position (de tout à fait d'accord à pas d'accord du tout). Entre les deux items, le $\mathrm{V}$ de Cramer est de 0.29 . Nous retenons ici tous ceux qui sont tout à fait d'accord ou d'accord avec l'un des deux items.

43. L'indicateur invite à choisir entre 5 positions (de tout à fait d'accord à pas du tout d'accord) par rapport à l'item «Pensez-vous que les personnes convaincues de meurtre devraient être condamnées à la peine de mort ». Il s'agit d'une question optionnelle. Nous retenons ici les deux positions favorables à la peine de mort.

44. La distinction entre sans religion croyants et non croyants fait ici sens, les premiers étant proches des catholiques pratiquants irréguliers ou non pratiquants.

45. Là encore, le fait d'avoir quelques croyances religieuses fait baisser chez les sans religion le pourcentage d'identité de gauche.

46. Cf. Bruno CAUTRÈs, Christine PINA, Kristoff TALIN, «L'influence de la religion sur les attitudes politiques: essai d'analyse spatio-temporelle dans l'Union européenne », in Pierre BRÉCHON et Bruno CAUTRÈs (éds.), Les enquêtes Eurobaromètres. Analyse comparée des données socio-politiques, Paris, L'Harmattan, 1998, pp. 269-286 (coll. «Logiques politiques») et Pierre BRÉCHON, «Les Européens et la politique », Futuribles n ${ }^{\circ}$ spécial 200, pp. 63-84. Les enquêtes électorales françaises montrent que la relation entre le vote et l'intégration au catholicisme faiblit un peu, surtout du fait du moindre positionnement des sans religion à gauche, les catholiques restant toujours très fortement marqués à droite. Cf. Pierre BRÉcHON, «Is There Religious Voting in France ? Political Tradition and 1997 Législative Election ", La Lettre de la Maison française d'Oxford, n 8, 1997, pp. 5-29.

47. Contrairement à d'autres enquêtes, l'intégration au catholicisme ne semble pas ici immuniser contre le Front national. 


\section{RÉSUMÉS}

Analysant les données de l'enquête ISSP en France, l'auteur montre que les tendances de long terme à la sécularisation continuent à faire sentir leurs effets. Les images des religions sont en demi-teinte, l'affaiblissement du catholicisme se poursuit, le système des croyances chrétiennes s'effiloche, des recompositions de croyances se font jour, la croyance en une vie après la mort n'étant plus toujours liée à une vision chrétienne. Des croyances parallèles autour de la maîtrise du futur semblent aussi se développer et toucher tout particulièrement les catholiques un peu décalés par rapport à leur institution. Le religieux est moins régulé par le catholicisme mais il n'y a pas de véritable émergence d'un religieux nouveau systématisé. Les attitudes des Français sont encore largement construites autour du couple catholicisme-irréligion. Et la montée de l'irréligion explique largement le déclin des valeurs traditionnelles.

Using data from the ISSP survey in France, the author shows that the long-term secularization trends are still being felt. The images of religions are blurred; the weakening of Catholicism is continuing; the system of Christian beliefs is gradually coming apart; recompositions of beliefs are under way, the belief in life after death being no longer always associated with a Christian vision. Parallel beliefs about the understanding of the future also seem to be developing and they are affecting in particular Catholics who are slightly at odds with their institution. The realm of religion is less regulated by Catholicism but one does not see a new systematized religious system emerging. The attitudes of the French still remain markedly organized around the couple Catholicism-irreligion, and the increase of irreligion explains for the most part the decline of traditional values.

Analizando los datos de la encuesta ISSP en Francia, el autor muestra que las tendencias de largo plazo a la secularizatión siguen produciendo efectos. Las imágenes de las religiones son difusas, el debilitamiento del catolicismo prosigue, el sistema de las creencias cristianas continua debilitándose, varias recomposiciones de las creencias aparecen, la creencia en una vida despues de la muerte no siendo necesariamente relacionada con una vision cristiana. Se desarrollan aparentemente creencias paralelas respecto al manejo del futuro, particularmente entre católicos que están un poco desconectados con su institution. Lo religioso está menos regulado por el catolicismo pero no existe verdadera conformación de un nuevo 'religioso sistematizado'. Las actitudes de los franceses están todavía ampliamente construidas alrededor de la pareja catolicismo-irreligion. Y el aumento de la irreligión explica en gran medida el ocaso de los valores tradicionales.

\section{AUTEUR}

\section{PIERRE BRÉCHON}

Institut d'Études Politiques de Grenoble, Centre d'informatisation des données socio-politiques 